\title{
HUGO LECTEUR DE BYRON: UN CONTRE-SENS VOLONTAIRE*
}

\author{
Bruno Sibona**
}

\begin{abstract}
Résumé: Il s'agit dans cet article du complexe système d'agrafes qui relie le poème de Victor Hugo 'Mazeppa', extrait de son recueil Les Orientales, au conte oriental de Lord Byron du même nom, qui a inspiré le jeune Hugo, et à sa traduction par Amédée Pichot, Hugo n'ayant pu lire le texte anglais. J'utiliserais pour ce faire une méthodologie intertextuelle et comparative inspirée par louvrage de Harold Bloom, The Anxiety of Influence et les outils théoriques de Gilles Deleuze et Félix Guattari tels qu'ils sont exposés dans Mille Plateaux.
\end{abstract}

Ivan Stépanovitch Mazeppa (1644-1709), hetman des cosaques, héros national ukrainien du $17^{\mathrm{E}}$ siècle, a été à plusieurs reprises une source d'inspiration pour les artistes. En 1818, durant un séjour à Venise, dans un long poème épique inspiré par un récit de Voltaire, Byron fait le récit d'un épisode de jeunesse qui aurait pu être fatal à Mazeppa. Cette aventure fut paradoxalement la cause initiale de son élévation:

Mazeppa, alors page du roi de Pologne Jean Casimir V, menait à la cour une vie fort dissolue jusqu'au jour où, surpris

\footnotetext{
' Recebido para publicação em novembro de 2004.

" Professor da City of London School, Londres.
} 
en flagrant délit d'adultère par un noble polonais, il fut attaché nu et enduit de goudron sur un cheval sauvage et abandonné à la course furieuse de l'animal. Le cheval, né dans les déserts de l'Ukraine, y transporta Mazeppa exténué de fatigue et de faim qui fut recueilli par quelques paysans. La reconnaissance le fixa parmi ses libérateurs dont il partagea la vie inquiète et belliqueuse. Plus tard, ses qualités d'homme d'action doublées d'une intelligence subtile et d'une vaste culture le firent élire hetman, c'est-à-dire chef des cosaques d'Ukraine, très apprécié du jeune tsar Pierre le Grand. Par une tragique erreur de jugement, Mazeppa s'allia au roi de Suède, Charles XII, dans sa guerre contre le tsar et ce retournement d'alliance consomma sa perte. Byron nous le montre en vieux général vaincu essayant de distraire le roi épuisé en lui racontant l'histoire de sa jeunesse. Le roi s'endort, et Byron de conclure: 'What mortal his own doom may guess?' ('Quel mortel peut son destin deviner?') A la lecture du texte de Byron traduit par Amédée Pichot en 1821, Hugo s'empare du thème et compose un poème divisé en deux parties pour ses Orientales. La première décrivant la course proprement dite dans toute son horreur et sa férocité fantasmatique, la deuxième ajoutant un commentaire dans lequel il compare Mazeppa au poète banni du commun des mortels pour ses excentricités, attaché sur le cheval fou de son inspiration. Mais pour finir, c'est ce voyage à travers les délires et souffrances du génie qui lui apporte la gloire: 'Sa sauvage grandeur naîtra de son supplice'.

Cette courte étude porte essentiellement sur deux aspects de la relation intertextuelle Byron-Hugo. A l'aide d'une problématique proche de celle dégagée par Harold Bloom dans son livre The Anxiety of Influence, je commencerai par examiner la citation de Byron mise en exergue par Hugo en tête de son poème et qui fonctionne comme une prétendue reconnaissance de dette 
envers le poète anglais, alors que c'est avec le traducteur qu'Hugo entretient des relations intertextuelles beaucoup plus cruciales. Je montrerai ensuite comment les deux volets, l'un grotesque et l'autre sublime, du texte hugolien représentent deux embranchements distincts par rapport au poème de Byron, le second volet sublime étant un commentaire métapoétique à la fois du premier volet grotesque et du texte byronnien.

\section{Le passage d'un texte à l'autre est assuré par une erreur de traduction.}

'Away! Away !' crie le narrateur à la fin du $9^{\mathrm{e}}$ chant du Mazeppa de Byron afin d'exprimer la soudaineté et la furie caractérisant le début de la course folle. 'Away! Away !' répètet-il au début du $10^{\mathrm{e}}$ chant, et une fois de plus trois vers plus loin. L'écho de ces mots résonne encore en deux autres occasions au début et au milieu du $11^{\mathrm{e}}$ chant. La répétition de l'impératif liée à l'exclamation renforce la narration, emportant littéralement le lecteur ou l'auditeur au loin, capturant son attention dans une dynamique chaîne de son. En répétant cinq fois le double cri, Byron utilise une technique de récitation qui appartient typiquement au genre épique et qui nous implique dans le rythme du galop de la chevauchée qui commence.

Nous savons de sources variées que Hugo ne lisait ni ne comprenait l'anglais. Il a lu Byron dans la traduction d'Amédée Pichot. Or, dans cette traduction, le mot 'away', qui aurait pu être traduit simplement par 'au loin', n'apparaît pas ainsi. A la fin du 11e chant de la version Pichot, au début du 12e et ensuite à l'imparfait dans le 13e, nous trouvons : 'nous volons'. Une telle métaphore tout à fait absente du texte de Byron est typique du romantisme prosaïque de Pichot qui tend à systématiquement émousser le style acéré du poète anglais. 
Hugo était tout à fait conscient de ces problèmes d'affaiblissement du texte original et guère satisfait de la traduction Pichot. Il a certainement essayé de se référer au texte de Byron en dépit de sa propre incapacité à lire l'anglais puisque les mots : 'Away! Away!' figurent en anglais comme épigraphe de son propre poème, après la dédicace à son ami, le peintre Louis Boulanger qui avait déjà illustré l'histoire de Mazeppa dans deux tableaux. Hugo révèle ainsi la double source de son inspiration, visuelle et textuelle, mais dénie cette valeur inspirationnelle à la version française de Pichot ${ }^{1}$.

Dans ses Orientales, Hugo a entrepris une nouvelle interprétation du mythe de Mazeppa plutôt qu'une translitération du conte de Byron qui aurait de toute façon été impossible compte-tenu de la 'force' de Hugo telle qu'elle est définie par Harold Bloom ${ }^{2}$. Le Mazeppa de Hugo allait être un contre-sens de lecture volontaire du Mazeppa de Byron. Ceci est clairement démontré par l'appropriation (et la déformation) de la double exclamation que Hugo traduit dans son épigraphe par 'En avant ! En avant !'3. L'idée de distance, d'espacement, a disparu au profit de l'idée de mouvement.

Par ailleurs, au niveau rhétorique, Hugo s'approprie les contre-sens du traducteur Pichot. Dans le texte du poète anglais,

\footnotetext{
' Ceci n'est guère surprenant lorsqu'on se rappelle ce qu'Hugo avait dit au sujet de la traduction contemporaine de Shakespeare en français qu'il préférait ne pas lire du tout de peur de lire du Letourneur à la place. Une telle remarque montre bien la méfiance d'Hugo à l'égard de toute tentative de traduction poétique.

${ }^{2}$ Bloom, Anxiety Influence: 'Strong poets make that history by misreading one another, so as to clear imaginative spaces for themselves', p. 5, and '[...] for really strong poets can read only themselves', p. 19.

${ }^{3}$ On retrouve ici le thème hugolien de la force qui va telle que l'a révélée Baudoin dans son analyse psychocritique.
} 
il est certes fait plusieurs fois mention du vent produit par la vitesse de la course et au moins une fois apparaissent des ailes d'oiseaux. Ce lien est à la fois métaphorique et métonymique mais, au contraire du poème de Hugo dans lequel la figure du vol est développée jusqu'à former une allégorie visionnaire globale, nulle part Byron ne dit que Mazeppa et son étalon 'volent'. Byron se montre en général parcimonieux dans son usage de la métaphore et c'est par conséquent à partir de l'amplification - ou du contre-sens - métaphorique de Pichot ('Nous volons') qu'Hugo peut se permettre de développer sa chaîne textuelle. Hugo accepte l'héritage (sans toutefois l'assumer ouvertement) et le développe par la métaphore filée du cheval ailé qui 'court' tout au long de son poème pour finir par aboutir au résultat poétique le plus évident : l'allégorie de Pégase.

De Byron à Pichot, puis de Pichot à Hugo, nous assistons donc à un renforcement de la fonction figurative dans le texte, renforcement qui va fournir le terrain sémantique et rhétorique au commentaire métapoétique qui suit dans la seconde partie du poème d'Hugo.

\section{L'omission de Pichot et l'appropriation de Byron: un double déni d'influence.}

Du point de vue de Hugo - 'poète fort' -, Byron n'était pas allé 'jusqu'à un certain point' ou avait 'échoué en n'allant pas assez loin', pour reprendre des expressions bloomiennes. Le Mazeppa de Hugo est né d'un possible désenchantement à la lecture du poème de son prédécesseur. Il s'est donc mis à écrire sa propre version dans laquelle on voit qu'il récupère et 'reconstruit' Byron, ou plutôt l'image distordue du poète anglais, à seule fin de construire sa propre personnalité créatrice. Par rapport à son prédécesseur, Hugo agit en prédateur; la figure 
de Byron et ses contes orientaux deviennent rapidement des éléments de son propre contexte d'auteur.

Dans ce même mouvement, Hugo reconnaît le fait que son texte, et par conséquent son inspiration, s'inscrit dans une chaîne textuelle pré-existante. Mais dans ce processus un maillon a disparu : Hugo a soigneusement évité toute référence explicite à la traduction de Pichot. Quelle que soit la qualité littéraire (ou plutôt son manque de qualité si l'on en croit à la fois Byron et Hugo) de ce chaînon manquant, la signification de son omission doit être pleinement appréciée. Une re-lecture de la traduction Pichot nous prouve qu'effectivement Hugo en a retiré une majeure partie de son inspiration, notamment pour sa première partie, dans laquelle il s'agit souvent d'une véritable ré-écriture versifiée.

En réalité, l'interprétation hugolienne du texte de Pichot fonctionne non seulement par amplification hyperbolique et accroissement de la figuralité, mais aussi, dans une certaine mesure, par déplacements et condensations comme dans le travail du rêve décrit par Freud ${ }^{4}$. Il est donc possible de considérer

'Sigmund Freud, chap.VI 'The Dream-work', in The Interpretation of Dreams, trans. by James Strachley (London: Penguin Books, 1976): 'Each elements of the dream's content turns out to have been "overdetermined" [...]. Not only are the elements of a dream determined by the dream-thoughts many times over, but the individual dream-thoughts are represented in the dream by several elements $[\ldots .$.$] . The elements of the dream are constructed out of the whole mass of dream.$ thoughts and each one of these elements is shown to have been determined many times over in relation to the dream-thoughts', pp. 388-389. Also, 'It thus seems plausible to suppose that in the dream-work a psychical force is operating which on the one hand strips the elements which have high psychical value of their intensity, and on the other hand, by means of overdetermination, creates from elements of low psychical value new values, which afterwards find their way into the dream-content. If that is so, a transference and displacement of psycbical intensities occurs in the process of dream-formation, and it is a result of these 
cette activité et ces techniques d'appropriation, de transformations, de condensations comme très proches de ce que Bloom appelle clinamen et tesser $a^{5}$.

Estève nous montre comment Hugo a utilisé le texte de Pichot:

Tout cela Victor Hugo l'a pris à son modèle, mais il le resserre à sa guise, le réfracte à travers son imagination, l'accommode à son dessein. L'interprétation symbolique lui appartient en propre. Elle a surgi à ses yeux sous la forme antithétique, entre les lignes de la traduction Pichot [...]. Il a ajouté au texte du poète anglais dans le premier cas [Mazeppa] une idée [...]. En imitant, il invente ${ }^{6}$. (c'est moi qui souligne)

On voit dans cette citation une certaine confusion entre le texte original de Byron et la traduction de Pichot. La distinction

that the difference between the text of the dream-content and that of the dreamthoughts comes about $[. .$.$] . The consequence of the displacement is that the dream-$ content no longer resembles the core of the dream-thoughts and that the dream gives no more than a distortion of the dream-wish which exists in the unconscious', p. 417.

5 '1. Clinamen, which is poetic misreading or misprision proper [...]. A poet swerves away from his precursor, by reading his precursor's poem as to execute a clinamen in a relation to it. This appears as a corrective movement in his own poem, which implies that the precursor poem went accurately up to a certain point, but then should have swerved, precisely in the direction that the new poem moves. 2. Tessera, which is completion and antithesis; I take the word not from mosaic-making, where it is still used, but from the ancient mystery cults, where it meant a token of recognition [...]. A poet antithetically "completes" his precursor, by so reading the parent-poem as to retain its terms but to mean them in another sense, as though the precursor had failed to go far enough', Bloom, Anxiety Influence, p. 14

" Estève, Byron, p. 304. 
n'est pas clairement établie entre ce qui revient à l'un et à l'autre dans l'influence exercée sur Hugo. Notre tâche est maintenant d'arriver à lire entre ces lignes, ou, plus précisément, entre celles du texte original anglais, celles de sa traduction en français et celles de la ou des versions de Hugo. Estève a remarqué que Hugo a pris modèle sur le texte de Pichot mais qu'il le 'resserre à sa guise, le réfracte à travers son imagination, l'accommode à son dessein'. Ce 'resserrement' et cette 'réfraction' sont similaires aux mécanismes de condensation et de déplacement actifs dans le travail du rêve, mais cette fois bien sûr au niveau intertextuel' ${ }^{7}$. Le texte du prédécesseur est ainsi analogue au stock d'images et de symboles inconscients qui nourrissent les rêvies du sujet ${ }^{8}$. L' 'accommodement' peut être observé dans les nombreux développements hyperboliques qu'opère Hugo sur certains motifs particuliers.

\section{IIII Le 'dépliement' des couches textuelles.}

J'aimerais maintenant approfondir l'analyse du système d'accroches qui relie les textes les uns aux autres. Lorsque nous commençons à lire le poème d'Hugo, nous sommes immédiatement frappés par le premier mot du texte lui-même, l'anacoluthe 'Ainsi'. Ce mot fonctionne comme une marque de reconnaissance d'un texte précédent (ou également d'un tableau, ne l'oublions pas). Cet adverbe représente pour le lecteur le

\footnotetext{
${ }^{7}$ Son inspiration plus personnelle interviendrait ainsi en partie comme un prisme distordant, le type de phénomène qu'à la suite du critique Jean-Pierre Richard j'aimerais appeler 'diffraction' ou 'téverbération'.

"Ce parallélisme me paraît par ailleurs légitimé par la comparaison extensive entre les processus du rêve et ceux de la création poétique qu'a établi Hugo luimême et qu'il a décrit de manière plus approfondie dans son texte Promontorium somnii.
} 
bord même de la tessera tandis qu'en même temps il autorise Hugo à libérer son propre texte des événements qui précédent le début de la folle chevauchée dans le conte de Byron. Cet 'Ainsi' nous place donc immédiatement et de plain-pied dans le véritable lieu symbolique où tout se joue. En corollaire, un blanc est laissé à remplir au lecteur qui doit lire la version Byron/Pichot s'il veut comprendre comment Mazeppa en est arrivé là. Bien sûr, la vaste majorité des lecteurs français contemporains connaissaient la traduction de Byron par Pichot (le plus grand succès d'édition de la première moitié du $19^{\mathrm{C}}$ siècle), ou étaient familiers de l'histoire grâce aux nombreuses représentations picturales et au spectacle du cirque équestre Franconi. De manière discrète mais efficace, ce vide référentiel fonctionne donc comme une autre marque de reconnaissance, de la part de Hugo, de son inscription dans une chaîne textuelle qui lui pré-existe.

Le poème de Hugo est composé de deux parties. La première, la plus longue, formée de 17 strophes, suit assez fidèlement la narration de la chevauchée frénétique qui commence au chant 9 chez Byron et au chant 11 chez Pichot'. Seules les deux dernières strophes de la première partie de Hugo font écho au commentaire byronien sur la difficulté de déchiffrer la destinée humaine.

La seconde partie du poème, avec seulement six strophes, propose un commentaire métapoétique de la première partie. De manière tout à fait frappante, cette seconde partie commence également avec l'adverbe 'Ainsi' qui fonctionne maintenant comme référence anaphorique à la première partie. Tout à fait intentionnellement, les trois premières lignes de la seconde partie se présentent donc comme des anamorphoses syntactiques et sémantiques des trois premières lignes de la première :

\footnotetext{
'Effectivement, Pichot n'a pas respecté le découpage en chants de Byron.
} 
$1^{\mathrm{c}}$ Ainsi, quand Mazeppa, qui rugit et qui pleure, A vu ses bras, ses pieds, ses flancs qu'un sabre effleure, Tous ses membres liés $[\ldots]$

$2^{\mathrm{c}}$ Ainsi lorsqu'un mortel, sur qui son dieu s'étale S'est vu lié vivant sur ta croupe fatale, Génie ardent coursier $[\ldots]$

Le contenu métapoétique est induit par un seul mot, 'génie', dont on connaît l'importance pour Hugo et qui représente le véritable (et double) clinamen entre la première partie narrative et son commentaire, mais aussi entre l'interprétation explicite de Byron sur l'histoire de Mazeppa et la lecture personnelle qu'en fait Hugo. Par conséquent, ce n'est plus trois versions de l'histoire de Mazeppa que nous devons comparer et mettre en perspective, mais bien quatre! En effet, les deux parties du poème de Hugo fonctionnent sur deux modes d'interprétations différents du mythe textuel tel qu'il avait été transmis par Byron: d'abord une mise en scène fictionnelle, puis un commentaire poético-discursif. Ces deux versions hugoliennes doivent être examinées séparément.

Dans sa première partie grotesque, Hugo accorde autant d'importance à certains motifs présents dans la version Pichot, comme par exemple le passage initial avec les bourreaux qui correspond également au tableau de Boulanger appartenant à Hugo à l'époque. D'autres motifs sont mentionnés mais voient leur importance réduite, tel la traversée de la rivière, ou déplacés, tel le passage relatif aux chevaux sauvages. Cependant, le déplacement effectué par Hugo sur cette scène-ci, et le fait que ces chevaux maintenant suivent Mazeppa et son étalon au lieu d'assister à la mort de l'étalon, opère une condensation telle que nous l'avons formulée auparavant. Le rythme hypnotique 
du galop est aussi maintenu grâce à de nombreuses répétitions. La vision cosmique du ciel, du soleil globulaire, de la succession des nuits et des jours est soigneusement respectée. Enfin deux aspects importants sont considérablement développés dans de nouveaux groupements d'images :

Le premier est le double motif du liquide et du feu, déjà présent dans la version Byron-Pichot, mais fortement accentué chez Hugo, peut-être parce que ce motif revêt de puissantes connotations sexuelles, aux résonnances sado-masochistes.

Le deuxième, sans doute le plus important, est le thème du vol dérivé du 'Nous volons' de Pichot, alors qu'il était à peu près absent dans l'original de Byron. Hugo l'introduit à deux reprises dans sa première partie, avant d'en faire une exploitation systématique dans sa seconde. Il y devient d'ailleurs le thème dominant, couplé avec celui de la vision. Ici, nous voyons que Hugo a formé un thème supplémentaire à partir de deux éléments différents de la version Byron-Pichot qu'il a pu fondre grâce à son extraordinaire alchimie verbale. Il a lié ce motif de la vision, crucial pour la compréhension de sa propre poétique, au thème de la course à partir de la séquence verbale 'Ils vont', 'Ils volent'. Il opère ainsi une sorte de glissando phonique et sémantique tout au long de son poème. Dès le début de la première partie, Hugo place Mazeppa dans la position du témoin de son propre tourment à partir du sens de la vue : 'Mazeppa qui rugit et qui pleur/a vu [...]'. La vue est ainsi le sens premier à l'aide duquel le héros reste en contact avec la réalité. Immédiatement après l'essor initial de la course, exprimé par un fulgurant cri métonymique fusionnant tous les acteurs ensemble, les bourreaux, le cheval et Mazeppa luimême, 'Un cri part', Hugo introduit le thème du vol ; 'volent avec les vents'. Si le rythme de la course est donné par la répétition au début des strophes 4 et 5 de l'expression reprise 
de Pichot 'Ils vont', au début de la strophe 7, la formule 'il voit' lui fait écho, et c'est comme si l'homophonie 'vu', 'volent', 'vont', 'voit' exemplifiait l'équation de la vision au vol, du 'voir' au 'voler' à partir de l'action catalytique du 'va', de l"aller'.

La configuration voir-aller-voler s'établit donc comme le lien thématique majeur entre les deux parties du poème de Hugo, et nous pouvons maintenant mieux comprendre comment la deuxième partie sublime peut fonctionner en tant que diffraction à la fois de la première partie et de la version Byron-Pichot.

La diffraction de signification entre les deux parties est considérable. Cependant, la seconde partie fonctionne comme une image-miroir de la première. En tant que telle, elle en renforce la dimension symbolique. L'histoire initiale ne produirait aucune signification métapoétique sans sa contre-partie spéculaire et demeurerait purement imaginaire, une allégorie sans aucune clef pour l'interpréter. Grâce à cette structure binaire inscrite dans le tissu textuel de son poème, Hugo ajoute une nouvelle perspective à l'histoire telle qu'elle est narrée par Byron, développant et perpétuant ainsi le mythe littéraire de Mazeppa.

Resumo: Este trabalho trata do complexo sistema de relações entre o poema de Victor Hugo "Mazeppa", extraído da coletânea Les Orientales, ao conto oriental de Lord Byron de mesmo nome, o qual inspirou o jovem Hugo, e a sua tradução por Amédée Pichot, uma vez que Hugo não pôde ter lido o texto em inglês. Será utilizada a metodologia intertextual e comparativa inspirada pela obra de Harold Bloom, The Anxiety of Influence, bem como os conceitos teóricos de Gilles Deleuze e Félix Guattari expostos em Mille Plateaux.

\footnotetext{
${ }^{10}$ Et l'on peut bien entendu penser ici à la 'force qui va' de Charles Baudoin.
} 


\section{Referências bibliográficas}

BAUDOIN, Charles, Psychanalyse de Victor Hugo. Paris: Armand Colin, 1972.

BLOOM, Harold, The Anxiety of Influence, a Theory of Poetry. Oxford: Oxford University Press, 1973.

BYRON, George G., Lord, Oeuvres complètes, traduites de l'anglais par MM. A.[médée] P.[ichot] et E.[usèbe] D.[e] S.[alle], troisième édition, 15 vols. Paris: Ladvocat, 1821.

BYRON, George G., Lord, Works, ed. by Jerome J. McGann. Oxford: Oxford University Press, 1986.

ESTEVE, Edmond, Byron et le romantisme français. Paris: Boivin, 1907.

FREUD, Sigmund, The Interpretation of Dreams, in Complete Works, trans. by James Strachey, The Standard Edition, 24 vols. London: The Hogarth Press, 1955, IV

FREUD, Sigmund, The Interpretation of Dreams, transl. by James Strachey, 15 vols. London: Penguin Books/The Pelican Freud Library, 1976, IV

HUGO, Victor, Oeuvres complètes/ Critique, ed. par Jean-Pierre Reynaud. Paris: Laffont/Bouquins, 1985.

HUGO, Victor, Oeuvres poétiques I \& II, ed. par Pierre Albouy. Paris: Gallimard/Pléïade, 1967.

HUGO, Victor, Les Orientales, ed. par Pierre Albouy. Paris: Gallimard/NRF, 1964 .

RICHARD, Jean-Pierre, Poésie et profondeur, Paris : Seuil, 1955.

VOLTAIRE, Histoire de Charles XII, roi de Suède, in Oeuvres bistoriques, ed. par René Pomeau. Paris: Gallimard/Pléïade, 1957. 